\title{
Mitteilungen der DVSE
}

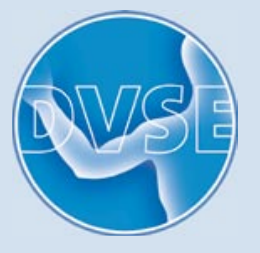

Obere Extremität 2009 · 4:116 DOI 10.1007/s11678-009-0029-5 C Springer -Verlag 2009

\section{Schulterwochen}

Im Rahmen der zertifizierten Fortbildung der Deutschen Vereinigung für Schulter- und Ellenbogenchirurgie e.V. (DVSE) ist ein 2-wöchiges Praktikum in einer von der DVSE anerkannten Ausbildungseinrichtung vorgesehen. Im 3. Quartal 2009 können folgende Kurse angeboten werden: Die Berücksichtung erfolgt in der Reihenfolge der Bewerbung. Voraussetzung ist die Mitgliedschaft in der DVSE.

- Roland-Klinik Bremen Zentrum für Schulterchirurgie

Arthroskopische Chirurgie und Sporttraumatologie Ausbilder:

Dr. Hans-Gerd Pieper 27.07. bis 31.07.2009

Teilnehmerzahl: 1

Anmeldung per E-mail: hpieper@roland-klinik.de
Deutsche Vereinigung für Schulterund Ellenbogenchirurgie (DVSE) e.V.

Präsident:

Prof. Dr. med. Markus Loew

Sektion für

Schulter- und Ellenbogenchirurgie

Orthopädische Universitätsklinik

Schlierbacher Landstraße 200 a

D-69118 Heidelberg

Tel. 062 21/96-62 24

Fax 062 21/96-83 88

markus.loew@ok.uni-heidelberg.de
- Vulpiusklinik GmbH

Bad Rappenau

Abteilung für Schulterund Ellenbogenchirurgie Ausbilder: Priv.-Doz. Dr. Wolfgang Pötzl

06.07. bis 18.07.2009

20.07. bis 24.07.2009

Teilnehmerzahl: 1

Anmeldung per E-mail: poetzl@vulpiusklinik.de

- Orthopädische Praxisklinik Münster

Ausbilder:

Prof. Dr. Jörn Steinbeck und Dr. Kai-Axel Witt 06.07. bis 18.07.2009 Teilnehmerzahl: 2 Anmeldung per E-mail: kontakt@oppk.de

- Klinik Fleetinsel Hamburg Schulter- und Ellenbogenchirurgie

Ausbilder:

Priv.-Doz. Dr. Achim

Hedtmann und Priv.-Doz.

Dr. Andreas Werner

Termin nach Absprache Teilnehmerzahl: begrenzt auf max. 2 Teilnehmer Anmeldung per E-Mail: werner@klinik-fleetinsel.de
Krankenhaus Agatharied

Hausham

Abteilung für Schulter-, Ellenbogen- und Handchirurgie Ausbilder:

Prof. Dr. Ulrich H. Brunner

06.07. bis 10.07.2009

Teilnehmerzahl: 1

Anmeldung per E-mail:

khagatharied.de
Margarete.Heiler@

\author{
Anmeldung per E-mail: \\ anja.hoefer@ \\ ok.uni-heidelberg.de \\ ATOS-Klinik Heidelberg \\ Schulter- und Ellenbogen- \\ chirurgie \\ Ausbilder: \\ Prof. Dr. Peter Habermeyer \\ und Dr. Sven Lichtenberg \\ Termin nach Absprache \\ Teilnehmerzahl: 2 \\ Anmeldung per E-mail: \\ habermeyer@atos.de
}

_ Orthopädische Klinik

König-Ludwig-Haus

Lehrstuhl für Orthopädie der Universität Würzburg Ausbilder:

Prof. Dr. Frank Gohlke 03.08. bis 21.08.2009

Teilnehmerzahl: 2 Anmeldung per E-mail: k-liebetruth.klh@ uni-wuerzburg.de

-Orthopädisch-Unfallchirurgische Praxis

Jansen/Raab/Böhm, Würzburg Ausbilder:

Priv.-Doz. Dr. Dirk Böhm

Termin nach Absprache

Teilnehmerzahl: 1

Anmeldung per E-mail:

boehm@ortho-wuerzburg.de

- OCM-Klinik München

Ausbilder:

Prof. Dr. Ernst Wiedemann

Termin nach Absprache

Teilnehmerzahl: 1

Anmeldung per E-mail:

Ernst.Wiedemann@gmx.de 